\title{
Unique Transcriptome and Gene Expression Analysis of Rice Seedling Reveals Different Cadmium Response Regulatory Mechanisms Between Indica and Japonica Rice
}

\section{Shan Tong}

Jiangxi Agricultural University

Xin Luo

Jiangxi Agricultural University

Hira Khanzada

Jiangxi Agricultural University

Ghulam Mustafa Wassan

Jiangxi Agricultural University

Licao Cui

Jiangxi Agricultural University

Guangliang Wu

Jiangxi Agricultural University

Jingai Tan

Jiangxi Agricultural University

Shilei Liu

Jiangxi Agricultural University

\section{Caijin Li}

Jiangxi Agricultural University

\section{Peng Wang}

Jiangxi Agricultural University

Xiangyu Zhang

Jiangxi Agricultural University

Jie Xu

Jiangxi Agricultural University

Haohua He

Jiangxi Agricultural University

Jianmin Bian ( $\sim$ jmbian81@126.com )

Jiangxi Agricultural University https://orcid.org/0000-0003-3703-379X 
Research article

Keywords: rice, Cd stress, seedling, transcriptome analysis, indica, japonica

Posted Date: September 8th, 2020

DOI: https://doi.org/10.21203/rs.3.rs-67623/v1

License: (c) (i) This work is licensed under a Creative Commons Attribution 4.0 International License. Read Full License 


\section{Abstract}

Background: In general, the Cd content in indica rice is usually higher than that in japonica. However, the mechanism for this discrepancy is unclear. Thus, understanding the genetic and molecular basis of $\mathrm{Cd}$ stress between indica and japonica is extremely important for rice improvement programs.

Results: In this study, two varieties of rice, japonica 02428 and indica CH891, were continuously exposed to $\mathrm{Cd}$ and seedlings of the two varieties at two critical stages ( $3^{\text {rd }}$ and $5^{\text {th }}$ day) were selected for the dynamic genes analysis by transcriptome method. The results showed that $\mathrm{CH} 891$ was more sensitive to Cd than 02428, and a total of 7,204 and 6,670 differently expressed genes (DEGs) associated with Cd stress were detected at $3^{\text {rd }}$ day and $5^{\text {th }}$ day. Furthermore, we divided these DEGs into three categories: SCR (sensitive variety with Cd-responsive), RCR (resistant variety with Cd-responsive) and CCR (common Cd-responsive). The enriched metabolic pathways analysis of DEGs preferentially expressed in a stagespecific and cultivars-specific manner, and secondary metabolic processes were enriched in SCR while protein metabolism and plant hormone were enriched in RCR. The diverted metabolic pathways might be the major cause for different response mechanism of $\mathrm{Cd}$ in indica and japonica rice.

Conclusion: These results provide a novel insight into the $\mathrm{Cd}$ response mechanism in rice seedlings between different varieties, and these important Cd-responsive DEGs were frequently involved in specific biological processes and metabolic pathways that might provide a novel insight over indica and japonica rice $\mathrm{Cd}$ response mechanism difference.

\section{Background}

Cadmium (Cd) is a heavy metal with high biotoxicity, which is toxic to crops and poses a potential threat for human health through food chain [1]. Low concentration of $\mathrm{Cd}$ in agricultural soil can be absorbed by crops [2]. However, the $\mathrm{Cd}$ stored in plants edible parts can contribute to considerable Cd intake in the human diet [3]. In human, there are many health issues caused by $\mathrm{Cd}$ accumulation, such as anemia, cancer, hypertension, cardiac failure, cerebrovascular infarction, emphysema, proteinuria, dysfunction in lungs and renal, cataract, and Itai-Itai disease [4-6].

Rice (Oryza sativa), an extremely important food crop, feeds about half of the world's population and is a major source of human daily intake. In some areas of China, Thailand, America and Japan, production of high Cd-polluted rice and the chronic Cd toxicity in populations were reported [7-9]. Nevertheless, humans are facing serious problems with $\mathrm{Cd}$ accumulation in rice, the $\mathrm{Cd}$ intake in average diet of the population in China and Japan is exceeded the standard of FAO/WHO. For this motive, it is an urgent need to reduce $\mathrm{Cd}$ accumulation in rice. In order to reduce $\mathrm{Cd}$ accumulation, it is necessary to understand the molecular mechanism of $\mathrm{Cd}$ response in rice. And as two major subspecies of cultivated rice, indica and japonica evolved differently, showed different response to Cd stress [10]. In general, the Cd content in indica rice is usually higher than that in japonica rice and there exist different sensitivity to $\mathrm{Cd}$ in these two varieties of rice [10]. However, the mechanism for this discrepancy is unclear. Thus, understanding 
the genetic and molecular basis of Cd stress between indica and japonica is extremely important for rice improvement programs.

Recently, many studies have used different rice cultivars to study the Cd stress [11], and several genes were identified to be associated with $\mathrm{Cd}$ response, such as $\mathrm{P}_{1 \mathrm{~B}}$-ATPase genes, natural resistanceassociated macrophage protein genes, cation diffusion facilitator genes, ATP-binding cassette transporter and other low-affinity cation transporter genes [12-15]. However, it was confirmed that genes encoding GST, heat shock protein and cytochrome P450 were also strongly induced under Cd stress [16]. Although functional analyses of these individual genes are helpful for understanding the regulatory mechanisms of $\mathrm{Cd}$ response in rice, the genetic basis of mechanisms for different subspecies is not clearly identified. Therefore, a comprehensive understanding of molecular mechanisms regulating seed development of different subspecies in $\mathrm{Cd}$ stress is required to facilitate the development of new insight into $\mathrm{Cd}$ response mechanism. Access to transcriptome and next generation RNA sequencing (RNA-seq) technology provides an opportunity to reveal genetic diversity among various genotypes/cultivars under Cd stress.

To identify genetic and molecular basis of $\mathrm{Cd}$ stress between indica and japonica, an elite japonica variety, 02428, and an elite indica variety, $\mathrm{CH} 891$, were continuously treated with $\mathrm{Cd}$ stress, and the seedling of the two varieties at two critical stages were selected for the analysis of the dynamic gene by transcriptome method. In total, we identified 7204 and 6584 DEGs at 3rd and 5th day. To investigate the different $\mathrm{Cd}$ response mechanisms in different cultivars and stages, we study enriched metabolic pathways of the DEGs and found that the response mechanisms preferentially were in a stage-specific and cultivar-specific manner. The results highlighted a new insight for difference in Cd tolerance between indica and japonica.

\section{Methods}

\section{Plant materials}

Two rice (oryza sativa L.) varieties were used in the study. Changhui 891 (CH891), excellent indica restore line in south China, generated from Key laboratory of crop genetics and breeding, Jiangxi Agricultural University, Jiangxi province, China, whose variety rights number in China Rice Data Center is CNA20161213.9 [17]. 02428 is a wide affinity japonica variety, selected and bred by Institute of genetic physiology, Jiangsu academy of agricultural sciences, Jiangsu province, China. It is a hybrid of radiation offspring of crab rice in Yunnan province and radiation offspring of Jibang rice in Shanghai [18], also being collected in the China Rice Data Center.

\section{Plant growth conditions and treatments}

Rice seedling were grown in culture dishes. Briefly, 30 seeds were selected by getting rid of shriveled and empty seed and surface-disinfected by immersing in $4 \%$ solution of sodium hypochlorite for twenty minutes and drenched for three times with distilled water. Thirty germinative seeds were placed in each 
dish, and the treatment groups (Cd) were treated with $100 \mathrm{mg} / \mathrm{L} \mathrm{CdCl}_{2}$ while the control groups (CK) with distilled water. Seedling were maintained in the incubator at $28^{\circ} \mathrm{C} \pm 2$ for $16 / 8 \mathrm{~h} \mathrm{light/dark}$ condition. Appropriate $\mathrm{CdCl}_{2}$ solution and distilled water were added every day according to the requirement to avoid desiccation. Three biological replicates were arranged for each line.

\section{Samples harvest and phenotype characterization}

Seedling of two varieties (CH891 and 02428) were harvested and measured the seedling length of the population at the third day (Cd3 and CK3) and fifth day (Cd5 and CK5). Seedlings were wrapped with tin foil paper, immediately frozenin liquid nitrogen, and stored at $-80^{\circ} \mathrm{C}$. Three biological replications and three technical replications were performed for the measurement of seedling length.

\section{mRNA library construction and sequencing}

Total RNA was extracted for three biological and technical replicates in each of 3rd and 5th day after Cd stress (DAS) and 3rd and 5th day after control (DAC) using Trizol reagent (Invitrogen, CA, USA) following the manufacturer's procedure. The total RNA quantity and purity were analysis of Bioanalyzer 2100 and RNA 6000 Nano LabChip Kit (Agilent, CA, USA) with RIN number > 7.0. Approximately 10 ug of total RNA representing a specific adipose type was subjected to isolate Poly (A) mRNA with poly-T oligoattached magnetic beads (Invitrogen). Following purification, the mRNA is fragmented into smallpieces using divalent cations under elevated temperature. Then the cleaved RNA fragments werereverse-transcribed to create the final cDNA library in accordance with the protocol for the mRNASeqsample preparation kit (Illumina, San Diego, USA), the average insert size for the paired-endlibraries was 300 bp ( \pm 50 bp). And then we performed the paired-end sequencing on an IlluminaHiseq4000 at the (LC Sceiences,USA) following the vendor's recommended protocol.

\section{Normalization of gene expression levels and identification of differentially expressed genes}

Sequencing reads were mapped to the reference sequences. The mapped reads of each sample were assembled using StringTie. Then, all transcriptomes from samples were merged to reconstruct a comprehensive transcriptome using perl scripts. After the final transcriptome was generated, StringTie and edgeR was used to estimate the expression levels of all transcripts. StringTie was used to perform expression level for mRNAs by calculating fragments per kilobase of exon model per Million mapped reads (FPKM). The differentially expressed mRNAs and differentially expressed genes (DEGs) were selected with log2 (fold change) $>1$ or log2 (fold change) $<-1$ and with statistical significance $(p$ value $<$ $0.05)$ by $\mathrm{R}$ package.

\section{Quantitative real-time PCR (qRT-PCR) validation}

In order to validate the RNA-seq results, different expression patterns of several genes was confirmed by quantitative real-time RT-PCR (qRT-PCR). For qRT-PCR, $1 \mu \mathrm{g}$ of total RNA was used to synthesized cDNA using PrimeScript ${ }^{\mathrm{TM}} \mathrm{RT}$ reagent Kit (Perfect Real Time) (TaKaRa). The qRT-PCR was carried out using SYBR® Premix Ex Taq II (Tli RNaseH Plus; TAKARA BIO Inc., Shiga, Japan) and determined in LightCycler 
480 (Roche, Basel, Switzerland) according to the manufacturer's instructions. The qRT-PCR reactions were amplified for $95 \circ \mathrm{C}$ for $30 \mathrm{~s}$, followed by 40 cycles of $95 \circ \mathrm{C}$ for $5 \mathrm{~s}, 55 \circ \mathrm{C}$ for $30 \mathrm{~s}$ and $72 \circ \mathrm{C}$ for $30 \mathrm{~s}$. All reactions were performed with three independent biological replicates for each sample and three technical replicates for each biological replicate were analyzed. The relative gene expression was calculated by the software of ABI7500 Real-Time PCR System using the $2^{-\Delta \Delta C t}$ method.

\section{Functional annotation and GO and KEGG classification}

All expressed genes and significant genes were functional annotated against databases NR, KEGG, KOG, Pfam and Swiss prot, respectively. For the gene matched to multiple protein sequences, the protein with the highest similarity score was considered as the optimal annotation.

\section{Results}

\section{Phenotypic variation between $\mathrm{CH} 891$ and 02428 under Cd stress}

Following exposure of seedlings of rice strains $\mathrm{CH} 891$ and 02428 to $\mathrm{Cd}$, large phenotypic variations. The seedling lengths of $\mathrm{CH} 891$ and 02428 were shorter under $\mathrm{Cd}$ stress compared with the control condition (Fig. 1A-D). Furthermore, the seedling length of $\mathrm{CH} 891$ was significantly longer than 02428 in control condition, while there was no significant differences between the two genotypes with Cd stress (Fig. 1E, F). It indicated that $\mathrm{CH} 891$ was more sensitive to $\mathrm{Cd}$ than 02428 . The experiment carried out continuously for 7 days and found that the top two seedling length inhibition rate were on 4th and 6th day (Fig. 1G). It indicated that genes had higher expression in the 3rd and the 5th day than other days. Therefore, seedlings were harvested at the 3rd and 5th day and then sequenced RNAs.

\section{RNA sequencing of seedling transcriptome of the two genotypes}

A total of 1,070 million reads with average of 46 million reads per sample were generated. While the total of valid reads is 1,057 million and the average valid read per sample is 46 million. The ratio of Q20 for each was above 99\%, and the Q30 base percentage was above 95\% (Supplementary Table S1). Therefore, the quality of the data was very high and meet the requirements for further analysis. To confirm the accuracy and reproducibility of the RNA-Seq results, 16 genes were selected for qRT-PCR using the specific primers (Supplementary Table S2). The validation results for the 16 genes are shown in Fig. 5A, B. The qRT-PCR results were all consistent with the RNA-Seq data (Supplementary Table S2). To conclude our transcriptome sequencing results were credible.

\section{Identification of differentially expressed genes (DEGs)}


By comparing samples of the same rice cultivar in different conditions (control and stress) and different rice cultivar ( $\mathrm{CH} 891$ and 02428) in the same condition at different stages, we assigned four comparison groups at the two stages and eight comparison groups in total were obtained. And then selected DEGs in different comparision respectively by restricting pval $\leq 0.05$. It indicated that not only the cultivar but also the treatments and time points affect the gene expression level. The number of up-regulated and downregulated genes in each comparison group is shown in Fig. 3A.

Functional enrichment analysis was performed for all these DEGs (Supplementary Fig. S1). KEGG enrichment analysis showed that plant-pathogen interaction, phenylpropanoid biosynthesis, tryptophan metabolism, flavonoid biosynthesis and diterpenoid biosynthesis pathways were enriched in Cd3_CH891 vs. Cd3_02428. Plant-pathogen interaction, tryptophan metabolism, phenylpropanoid biosynthesis, flavonoid biosynthesis, diterpenoid biosynthesis and amino sugar and nucleotide sugar metabolism pathways were enriched in CK3_CH891 vs. CK3_02428. Starch and sucrose metabolism, phenylpropanoid biosynthesis, glycolysis, carbon metabolism, alpha-linolenic acid metabolism, fatty acid degradation and brassinosteroid biosynthesis pathways were enriched in Cd3_CH891 vs. CK3_CH891. Plant-pathogen interaction and plant hormone signal transduction pathways were enriched in Cd3_02428 vs. CK3_02428. On the 5th day, phenylpropanoid biosynthesis, glutathione metabolism, flavonoid biosynthesis, brassinosteroid biosynthesis, amino sugar and nucleotide sugar metabolism and ABC transporters pathways were enriched in Cd5_CH891 vs. Cd5_02428. Tryptophan metabolism, sesquiterpenoid and triterpenoid biosynthesis and phenylpropanoid biosynthesis pathways were enriched in CK5_CH891 vs. CK5_02428. Phenylpropanoid biosynthesis, glutathione metabolism, diterpenoid biosynthesis, brassinosteroid biosynthesis, alpha-linolenic acid metabolism and $A B C$ transporters pathways were enriched in Cd5_CH891 vs. CK5_CH891. Protein processing in endoplasmic reticulum, porphyrin and chlorophyll metabolism, photosynthesis, phenylpropanoid biosynthesis and circadian rhythm-plant pathways were enriched in Cd5_02428 vs. CK5_02428.

All of the results revealed that there were more up-regulated DEGs under Cd stress than those under control condition in $\mathrm{CH} 891$, while there were more down-regulated DEGs under Cd stress than those under control condition in 02428 on either the 3rd or 5th day. Moreover, there was a higher number of DEGs obtained for Cd stress in $\mathrm{CH} 891$ (Cd-sensitive cultivar) than those in 02428 (Cd-resistant cultivar).

\section{Classification of differentially expressed genes (DEGs)}

A total of 7204 unique DEGs were detected in four groups on the 3rd day and 6670 unique DEGs obtained on the 5th day, these DEGs divided into 15 subgroups (Fig. 3B, C). Excluding these DEGs of the groups (CK3_CH891 vs. CK3_02428 at the third day and the groups contained CK5_CH891 vs. CK5_02428 at the fifth day) irrelevant to $C d$ stress, the DEGs of the rest seven subgroups divided into three categories: genes from sensitive variety with Cd-responsive (SCR), genes from resistant variety with Cd-responsive (RCR), and common Cd-responsive (CCR) DEGs. There were 849, 676 and 770 DEGs in SCR, RCR and CCR at the 3rd day, respectively (Supplementary Table S3). Whereas 959, 592 and 1516 DEGs were in SCR, RCR and CCR at the 5th day, respectively (Supplementary Table S3). 
In addition, we selected important Cd-responsive (ICR) genes in the three categories with llog2 (fold change) $\mid \geq 2$ and FPKM $\geq 2$ in at least one group to select representative DEGs. At the 3rd day, there were 554 ICR genes were screened, among which 194 for SCR, 154 for RCR and 206 for CCR (Supplementary Table S3). Whereas a total of 941 ICR genes were screened at the 5th day, of these 389 for SCR, 216 for RCR and 336 for CCR (Supplementary Table S3). Subsequently, there were 17, 9 and 21 ICR genes were detected at the two stages in SCR, RCR and CCR, respectively (Supplementary Table S3).

\section{Gene functional annotation analysis of SCR}

Gene functional annotation analysis was conducted for ICR of SCR. Cytoplasm and DNA binding were especially enriched in SCR3, oxidation-reduction process and protein binding were particularly enriched in SCR5. Transcription factor activity, sequence-specific DNA binding regulation of transcription, DNAtemplated, integral component of membrane and plasma membrane were enriched in both SCR3 and SCR5 (Supplementary Fig. S2). In order to gain more biological information and regulatory network, the Kyoto Encyclopedia of Genes and Genomes (KEEG) enrichment pathways was performed for understanding the molecular mechanism of $\mathrm{Cd}$ response in rice seedlings. KEGG analysis results showed that, isoflavonoid biosynthesis, inositol phosphate metabolism, flavonoid biosynthesis, and anthocyanin biosynthesis pathways were enriched in SCR3 (Fig. 2A). While isoflavonoid biosynthesis and anthocyanin biosynthesis pathways were significantly enriched. One DEG involved in anthocyanin biosynthesis was down-regulated while one was up-regulated in Cd3_CH891. And two DEGs involved in isoflavonoid biosynthesis were up-regulated in Cd3_CH891. Phenylpropanoid biosynthesis, alpha-linolenic acid metabolism, linoleic acid metabolism, glutathione metabolism, anthocyanin biosynthesis, ABC transporters, brassinosteroid biosynthesis and diterpenoid biosynthesis pathways were enriched in SCR5 (Fig. 2B). However, phenylpropanoid biosynthesis, ABC transporters, brassinosteroid biosynthesis and diterpenoid biosynthesis pathways were four extremely enriched pathways in SCR5. Eight DEGs involved in ABC transporters were all up-regulated in Cd5_CH891. One DEG involved in phenylpropanoid biosynthesis was down-regulated while seventeen were up-regulated in Cd5_CH891. Five DEGs involved in brassinosteroid biosynthesis were all up-regulated, and six DEGs involved in diterpenoid biosynthesis were all up-regulated in Cd5_CH891. Interestingly, most of DEGs involved in enriched pathways were upregulated in $\mathrm{CH} 891$ under $\mathrm{Cd}$ stress.

\section{Gene functional annotation analysis of RCR}

Plasma membrane, integral component of membrane and extracellular region were enriched in ICR of RCR3 in the GO enrichment analysis, while cytoplasm and chloroplast were enriched in ICR of RCR5. Transcription factor activity, sequence-specific DNA binding protein binding, regulation of transcription and DNA-templated were enriched in both RCR3 and RCR5 (Supplementary Fig. S2).

In terms of KEGG pathway enrichment analysis, ribosome, regulation of autophagy and insulin resistance pathways were enriched in ICR of RCR3 (Fig. 2C). Among which ribosome and regulation of autophagy pathways were most enriched. One DEG involved in regulation of autophagy was down-regulated and the other two were up-regulated in Cd3_02428. One DEG involved in ribosome was up-regulated and six were 
down-regulated in Cd3_02428. Protein processing in endoplasmic reticulum, plant hormone signal transduction, thiamine metabolism, glucosinolate biosynthesis and base excision repair pathways were enriched in ICR of RCR5 (Fig. 2D). And protein processing in endoplasmic reticulum and plant hormone signal transduction pathways were most enriched. Four DEGs involved in protein processing in endoplasmic reticulum were up-regulated and other four were down-regulated in Cd5_02428. One DEG involved in plant hormone signal transduction was up-regulated and the other ten were down-regulated in Cd5_02428.

\section{Gene functional annotation analysis of CCR}

As far as ICR of CCR, chloroplast and zinc ion binding were enriched on the third day while ATP binding was enriched in the fifth day. And protein binding regulation of transcription, DNA-templated, cytoplasm, plasma membrane, integral component of membrane, transcription factor activity, sequence-specific DNA binding and DNA binding were enriched in both CCR3 and CCR5 (Supplementary Fig. S2).

In terms of KEGG pathway analysis, alpha-linolenic acid metabolism, phagosome, limonene and pinene degradation and fatty acid degradation pathways were enriched in CCR3 (Fig. 2E). Moreover, alphalinolenic acid metabolism and fatty acid degradation pathways were markedly enriched in CCR3. One DEG involved in alpha-linolenic acid metabolism was down-regulated and two DEGs were up-regulated. And one DEG involved in fatty acid degradation was up-regulated while the other one was downregulated. Plant-pathogen interaction, isoflavonoid biosynthesis, vitamin B6 metabolism, diterpenoid biosynthesis, and glutathione (GSH) metabolism pathways were enriched in the fifth day (Fig. 2F). Among which plant-pathogen interaction, isoflavonoid biosynthesis, and glutathione (GSH) metabolism pathways were observably enriched. One DEG involved in isoflavonoid biosynthesis was down-regulated, two were up-regulated. Two DEGs involved in glutathione metabolism were down-regulated, three were up-regulated. Seven DEGs involved in plant - pathogen interaction were down-regulated and twenty were up-regulated.

To understand the interaction of all the enriched GO terms at all the stages of SCR, RCR and CCR in the general, we constructed a network of significantly enriched GO terms (biological process) (Fig. 4). It showed that the biological process was focus on transport, response to different stimulus, metabolic of hormone, gene express, cellular activity, and secondary metabolic process. It suggested that different stress reactions were activated to respond to Cd stress.

\section{DEGs both in 3rd and 5th day after stress (DAS)}

In total, we obtained 46 common ICR in three categories at two stages and they were involved in several different pathways (Fig. 5C, D, E). Except for the pathways listed above, some other pathways were detected, such as inositol phosphate metabolism, cutin, suberine, and wax biosynthesis, biosynthesis of amino acids, starch and sucrose metabolism, ascorbate and aldarate metabolism, base excision repair, aminoacyl-tRNA biosynthesis, galactose metabolism, and circadian rhythm-plant. It indicated that not 
only the enriched pathways above but also insignificantly enriched pathways had interaction to regulate Cd stress in rice.

\section{Discussion}

\section{Genetic basis of $\mathrm{Cd}$ stress in rice}

The Cd stress extremely affects rice growth and development [19-20], such as photosynthesis, transpiration and other physiological processes of plants and produces excessive oxygen free radicals. In recent years, Cd has fascinated great attention due to its harmful effects on plant productivity. Several studies have shown that different cultivars showed different responses under Cd stress [11, 21]. However, the regulation mechanism between indica and japonica under $\mathrm{Cd}$ stress have not yet been reported. In the present study, we evaluated an elite japonica variety, 02428 (Cd sensitive cultivar), and one elite indica variety, $\mathrm{CH} 891$ (Cd resistant cultivar), by exposing seedling were continuously treated with $\mathrm{Cd}$ solution $100 \mathrm{mg} / \mathrm{L}$ for one week, seedling of the two varieties at two critical stages, when it determines the length of the seedling of the two varieties, were selected for the dynamic genes analysis under Cd stress by transcriptome method. The results showed that the metabolic pathways between 3rd DAS and 5th DAS were different not only for 02428 but also for $\mathrm{CH} 891$, implying that distinct genetic systems might be responsible for $\mathrm{Cd}$ stress at different stages in rice, a thorough and dynamic gene analysis for rice $\mathrm{Cd}$ stress was necessary. Otherwise, the metabolic pathways between $\mathrm{CH} 891$ and 02428 were also different at the same stage (3rd or 5th DAS), these metabolic pathways might be the cause that indica and japonica showed different response to $\mathrm{Cd}$ stress, therefore, the exploitation genes in these metabolic pathways might fully explain the differences in Cd stress between indica and japonica. All, the DEGs analysis showed that the difference in metabolic pathway not only exists in different genotypes but also in different stages.

\section{The stage specific expression of DEGs between indica and japonica}

KEGG pathway analysis was performed for all DEGs with significant differences. It showed that the enriched pathways of all DEGs are not significantly different from those of the following three categories (SCR, RCR, CCR), except for some amino sugar metabolism, amino acid metabolism, sugar metabolism and nucleotide sugar metabolism. In general, metabolic pathways of all DEGs mainly focus on basic life activities, secondary metabolic processes, plant-pathogen interaction and plant hormone signal transduction pathways. This suggests that the following analysis would not be too disturbed by the background.

To give more detailed information for the genes response to $C d$ stress in rice, we divided DEGs into three categories (SCR, RCR, and CCR) at the two critical stages. The enriched metabolic pathways analysis of DEGs indicated that these DEGs preferentially expressed in a stage-specific and cultivars-specific manner, some significant metabolic pathways were identified for SCR, RCR and CCR. 


\section{Expressed Gene special in SCR}

The isoflavonoid biosynthesis and anthocyanin biosynthesis pathways were enriched in SCR3. Isoflavones were found to play an important role in plant defense reactions [22]. Under Cd stress, the cell wall of rice was damaged, which made it susceptible to pathogen infection and then induced the expression of isoflavones to improve the disease resistance in plants [23]. Previous studies showed that isoflavones are usually abundant in legumes but low in other plants [24]. Combined with our result, it may provide new insight into the response of rice to $\mathrm{Cd}$ stress at the isoflavone level.

Moreover, Anthocyanin is a kind of antioxidant, which can scavenge oxygen free radicals in plant cells and relieve the toxicity of reactive oxygen [25]. Roychoudhury reported that anthocyanin biosynthesis was induced under $\mathrm{CdCl}_{2}$ [11], which was consistent with our result. It suggested that anthocyanin, as an antioxidant, relates to $\mathrm{Cd}$ stress response in rice and may relieve the toxicity of $\mathrm{Cd}$.

Phenylpropanoids biosynthesis was one of the enriched KEGG pathways in SCR5. Phenylpropanoids participated in the antioxidant activity of cell walls and the biosynthesis of lignin, which plays an important role in the response of plants to abiotic stress [26]. Several reserchers have found that phenylpropanoids metabolite was significantly altered by Cd stress in rice [27] and Arabidopsis helleri [28]. Our results confirmed that phenylpropanoids contribute to $\mathrm{Cd}$ response in rice. Besides, another enriched pathway in SCR5 was ABC transporters pathway, which was metabolic pathway authenticated to be important under $\mathrm{Cd}$ in rice [29]. Using the energy released by hydrolyzed ATP, ABC transporters can actively transport a variety of heavy metals through the cytomembrane, which is associated with plant stress tolerance and detoxification of heavy metals [30]. However, the result showed that $A B C$ transporters was found to be enriched in SCR5 may put forward that $A B C$ transporters participate in $C d$ response in rice and have possibility to inhibit $C d$ stress. From our results and previous study, $A B C$ transporters actually play important role in response to Cd stress. Diterpenoids, as secondary metabolites, was reported to inhibit intracellular ROS production and lipid peroxidation, and then enhanced the antioxidant defense system in Sideritis [31]. Beside, our study found that diterpenoid biosynthesis pathway was enriched in SCR5, it suggested that diterpenoid metabolism also be induced by Cd in rice and plays an important role in response to $\mathrm{Cd}$ stress in rice. Brassinosteroid (BR) has a wide range of biological functions and plays an important role in the adaptability of plants under stress. Bajguz and Hayat found that external application of BR can improve the salt tolerance of plants [32]. Our result indicated that BR was also related to $\mathrm{Cd}$ response in rice, and DEGs involved in this pathway were all related to cytochrome P450 and were up-regulated, which is consistent with the fact that cytochrome P450 participates in detoxification metabolism [16].

\section{Expressed Gene special in RCR}

In terms of RCR, the KEGG pathways were also different at 3rd and 5th DAS. Ribosome-related DEGs play key roles in cold stress signal transduction [33]. Therefore, ribosome pathway was identified to be enriched in this study. In addition to ribosome, the regulation of autophagy was another enriched pathway in RCR3. Autophagy can transport oxidative proteins and injured intracellular substances 
caused by stress into vacuoles for degradation, thereby reducing the accumulation of toxic substances in the cytoplasm [34]. It plays an important role in plant development and response to stress [35]. Thus, our study found that regulation of autophagy pathway was induced in RCR3 further verified that it played a role in Cd stress in rice to a certain extent, and these two pathways were newly identified in rice under $\mathrm{Cd}$ stress here, and which identified only at 3rd DAS.

As far as RCR5, plant hormone signal transduction was enriched. When in the unfavorable environment, plants must respond quickly and accurately to activate the necessary physiological responses which are usually mediated by plant hormones [36]. Phytohormones have been demonstrated to play an important role in mediating plant growth plasticity in response to metal stress include Cd [37]. Consistently, we detected plant hormone signaling transduction pathway in RCR5. Our result was consistent with the previous study in rice, however, it was only identified at RCR5, and protein processing in endoplasmic reticulum pathway was also enriched in RCR5. Cellular exposure to $\mathrm{Cd}$ is known as strongly induce the unfolded protein response, which suggests that the endoplasmic reticulum (ER) is preferentially damaged by $\mathrm{Cd}$ in yeast [38]. In our study, it was newly identified in rice under $\mathrm{Cd}$ stress. It suggested that $\mathrm{Cd}$ can lead to protein alteration among ER in rice.

\section{Expressed Gene special in CCR}

Differences also existed in CCR in the 3rd and 5th DAS. Alpha-linolenic acid metabolism and fatty acid degradation can be summarized as fatty acid metabolism, which was related to Cd response in CCR3. Previous studies revealed that heavy metal can specifically alter fatty acid metabolism in plants, Cd can enhance the level of lipid peroxides and lead to strong changes in fatty acid content in rice [39-40]. Fatty acid metabolism was found to be induced by heavy metal $\mathrm{Cr}$ in rice [41]. In this study, our findings provided further evidence that fatty acid metabolism is a stress response to $\mathrm{Cd}$ stress in rice. As far as the KEGG pathway analysis in CCR5, plant-pathogen interaction, GSH metabolism and isoflavonoid biosynthesis pathway were enriched. There is a very complex interaction between the endogenous bacteria or fungi in the plant. These microorganisms can produce a variety of biological effects in plants, they can form a special growth state to better adapt to the environment [42]. Several previous studies have found that plant pathogen interaction is induced by plant pathogen invasion [43]. The finding of this study puts forward the possibility that $\mathrm{Cd}$ damages the function and structure of cells and provides the conditions for pathogen attack. Many studies have reported that GSH alleviates heavy metal toxicity in rice $[28,41]$. However, the binding of GSH to heavy metal was catalyzed by glutathione S- transferase (GST), which is an important class of antioxidant and detoxification proteins in plant cells. In this study among the DEGs involved in GSH metabolism, all of the six were GST genes. It suggested that GST make a difference in GSH metabolism, which participates in Cd response in rice. Also, similar to pathway analysis in SCR3, isoflavonoid biosynthesis pathway reacted on Cd stress in CCR5. It implied that isoflavonoid biosynthesis pathway generally exist in Cd response in rice.

It can be observed that not only the response mechanisms between 3rd and 5th DAS in the same category but also mechanisms between different categories were different. Enriched pathways in SCR were associated with secondary metabolic processes while protein metabolism and plant hormone signal 
transduction were enriched in RCR. It indicated that $\mathrm{CH} 891$ might respond to $\mathrm{Cd}$ by inducing secondary metabolic processes and then can develop its immunity and resistance to adversity. Differently, the process of protein production and degradation was affected in 02428 , so as to protect itself from Cd. It may prove that the response mechanism of $\mathrm{Cd}$ in indica and japonica rice was different in one way.

\section{The common DEGs between 3rd and 5th DAS in indica and japonica}

With the exception of metabolic pathways discussed above, the common DEGs in three categories at two stages were detected, which were enriched in various pathways (Fig. 4C, D, E). Some pathways were discussed above and others were not significantly enriched in this study, which may involve the minor genes and conspire to Cd response in rice. Of these, DEGs, LOC_Os03g57240 (DST), a common DEG in RCR at 3rd and 5th DAS, was reported to be a zinc finger transcription factor and relate to DNA replication, which negatively regulates drought and salt tolerance of rice [44]. Therefore, it was detected in this study, which implied DST regulated Cd stress in rice. We selected four genes to conduct qRT-PCR, and they were verified compared with RNA-Seq (Fig. 2B).

\section{Conclusions}

In this study, high-throughput sequencing was used to sequence the rice seedling transcriptome treated with $\mathrm{Cd}$ at different stages, which highlighted the transcriptional variations among two different rice varieties under $\mathrm{Cd}$ conditions. Statistical analysis of 7204 and $6670 \mathrm{DEGs}$ revealed three categories for a total of 554 and 941 ICR DEGs in rice at two stages, respectively. Furthermore, these important Cdresponsive DEGs were frequently involved in specific biological processes and metabolic pathways that might provide a novel insight over indica and japonica rice $\mathrm{Cd}$ response mechanism difference.

\section{Abbreviations}

Cadmium (Cd), common Cd-responsive (CCR), day after Cd stress (DAS), day after control (DAC), differentially expressed genes (DEGs), fragments per kilobase of exon model per Million mapped reads (FPKM), Gene Ontology (GO), important Cd-responsive (ICR), Kyoto Encyclopedia of Genes and Genomes (KEEG), resistant variety with Cd-responsive (RCR), sensitive variety with Cd-responsive (SCR).

\section{Declarations}

\section{Acknowledgements}

We acknowledge support by Hangzhou Lianchuan Biotechnology Co. LTD. We also thank those who were not mentioned for their help with our manuscript.

\section{Funds}


This research was supported by grants (2016YFD0101801) from The National Key Research and Development Program of China and Technology Department and grants (20192BCB23010, 20192ACBL20017) from Project of Science and Technology Department of Jiangxi Province and grant (201910410003) from National Undergraduate Training Program for Innovation and Entrepreneurship and (GJJ170241) Science and technology research project of Jiangxi Provincial Department of Education.

\section{Availability of data and materials}

The data supporting the conclusions of this article are included within the article and its additional files.

\section{Author contributions}

S.T designed and performed experiments, analyzed data and wrote the manuscript. X.L participated in designing and performing experiment. H.K and G.M.W completed the manuscript with inputs in technical support, critical writing and suggestions regarding the manuscript. L.C, G.W, J.T, S.L, C.L, P.W, X. Z, J.X participated in performing experiments. H.H and J.B. conceived and supervised the experiments. All authors reviewed the manuscript.

\section{Ethics approval and consent to participate}

The rice line 02428 and $\mathrm{CH} 891$ (including the seeds) obtained from the native cultival line and all plants were grown in the test fields of Jiangxi Agriculture University. Sampling of plant materials were performed in compliance with institutional, national, and international guidelines.

\section{Competing interest}

The authors declare that they have no conflict of interest.

\section{References}

1. Wu FB, Zhang G. Genotypic Differences in Effect of Cd on Growth and Mineral Concentrations in Barley Seedlings. Bull Environ Contam Toxicol. 2002;69(2):219-27.

2. Moulis JM, Frank T. New perspectives in cadmium toxicity: an introduction. Biometals. 2010;23(5):763-8.

3. Wagner G. Accumulation of Cadmium in Crop Plants And Its Consequences to Human Health. Adv Agron. 1993;51:173-212.

4. Godt J, Scheidig F, Grosse-Siestrup C, et al. The toxicity of cadmium and resulting hazards for human health. Journal of Occupational Medicine Toxicology. 2006;1:22.

5. Satarug S, Baker JR, Urbenjapol S, et al. A global perspective on cadmium pollution and toxicity in non-occupationally exposed population. Toxicol Lett. 2003;137(1-2):65-83.

6. Yamagata N, Shigematsu I. Cadmium pollution in perspective. Bullinstpublic Health. 1970;19:1-27. 
7. Jin T, Nordberg M, Frech W, et al. Cadmium biomonitoring and renal dysfunction among a population environmentally exposed to cadmium from smelting in China (ChinaCad). Biometals. 2002;15(4):397-410.

8. Nordberg GF, Jin T, Kon Q, et al. Biological monitoring of cadmium exposure and renal effects in a population group residing in a polluted area in China. Sci Total Environ. 2001;199:111 - 114.

9. Ryumon $\mathrm{H}$, Witaya $\mathrm{S}$, Muneko $\mathrm{N}$, et al. Cadmium induced renal dysfunction among residents of rice farming area downstream from a zinc-mineralized belt in Thailand. Toxicol Lett. 2010;198:26-32.

10. Uraguchi S, Fujiwara T. Cadmium transport and tolerance in rice: perspectives for reducing grain cadmium accumulation. Rice. 2012;5:1-8.

11. Roychoudhury A, Basu S, Sengupta DN. Antioxidants and stress-related metabolites in the seedlings of two indica rice varieties exposed to cadmium chloride toxicity. Acta Physiol Plant. 2012;34:83547.

12. Yuan L, Yang S, Liu B, et al. Molecular characterization of a rice metal tolerance protein, OsMTP1. Plant Cell Rep. 2012;31(1):67-79.

13. Oda $\mathrm{K}$, Otani M, Uraguchi S, et al. Rice ABCG43 is Cd inducible and confers Cd tolerance on yeast. Bio Biotechnol Biochem. 2011;75(6):1211-3.

14. Uraguchi S, Kamiya T, Sakamoto T, et al. Low-affinity cation transporter (OsLCT1) regulates cadmium transport into rice grains. Proceedings of the National Academy of Sciences. 2011;108:2095920964.

15. Williams LE, Mills RF. P(1B)-ATPases-an ancient family of transition metal pumps with diverse functions in plants. Trends in Plant ence. 2005;10(10):491-502.

16. Ogawa I, Nakanishi $\mathrm{H}$, Mori S, et al. Time course analysis of gene regulation under cadmium stress in rice. Plant Soil. 2009;325(1-2):97.

17. Liu CY, Wan JM, Zhu CL, et al. The Mapping of Brown Planthopper Resistance Gene in Inidica Restorer Changhui 891. Acta Agriculturae Universitatis Jiangxiensis. 2016;38(2):232-7.

18. Wang ZM, Zou JS, Zheng WR, et al. Breeding in New Wide Compatibility Lines (WCL) of Japonica Rice. Hybrid Rice. 1990;(6):32-36.

19. Liu F, Liu X, Ding C, et al. The dynamic simulation of rice growth parameters under cadmium stress with the assimilation of multi-period spectral indices and crop model. Field Crops Research. 2015;183:225-34.

20. Song W. Variation of Cd concentration in various rice cultivars and derivation of cadmium toxicity thresholds for paddy soil by species-sensitivity distribution. J Integr Agric. 2015;14:1845-54.

21. Liu C, Mehdy MC. A nonclassical arabinogalactan protein gene highly expressed in vascular tissues, AGP31, is transcriptionally repressed by methyl jasmonic acid in Arabidopsis. Plant Physio. 2007;145:863-74.

22. Mortier V, Fenta BA, Martens C, et al. Search for nodulation-related CLE genes in the genome of Glycine max. J Exp Bot. 2011;62(8):2571-83. 
23. Chen Z. Increasing Tolerance to Ozone by Elevating Foliar Ascorbic Acid Confers Greater Protection against Ozone Than Increasing Avoidance. Plant Physiol. 2005;138:1673-89.

24. Kurzer M, Xu XJ. Dietary phytoestrogens. Annu Rev Nutr. 1996;17(17):353.

25. Neill SO, Gould KS, Kilmartin PA, et al. Antioxidant activities of red versus green leaves in Elatostema rugosum. Plant Cell Environment. 2010;25(4):539-47.

26. Bonawitz ND, Soltau WL, Blatchley MR, et al. REF4 and RFR1, Subunits of the Transcriptional Coregulatory Complex Mediator, Are Required for Phenylpropanoid Homeostasis in Arabidopsis. J Biol Chem. 2012;287(8):5434-45.

27. Chen L, Shi SL, Jiang NF, et al. Genome-wide analysis of long non-coding RNAs affecting roots development at an early stage in the rice response to cadmium stress. BMC Genom. 2018;19:460.

28. Corso M. Schvartzman, et al. Contrasting cadmium resistance strategies in two metallicolous populations of Arabidopsis halleri. New Phytol. 2018;218:283-97.

29. Shah K, Kumar RG, Verma S, et al. Effect of cadmium on lipid peroxidation, superoxide anion generation and activities of antioxidant enzymes in growing rice seedlings. Plant ence. 2001;161(6):1135-44.

30. Gaillard S, Jacquet H, Vavasseur A, et al. AtMRP6 / AtABCC6, an ATP-Binding Cassette transporter gene expressed during early steps of seedling development and up-regulated by cadmium in Arabidopsis thaliana. BMC Plant Biol. 2008;8(1):22-2.

31. González-Burgos E. Diterpenoids isolated from Sideritis species protect astrocytes against oxidative stress via Nrf2. Journal of natural products. 2012;75:1750-8.

32. Bajguz A, Hayat S. Effects of brassinosteroids on the plant responses to environmental stresses. Plant Physiology Biochemistry. 2009;47(1):1-8.

33. Bai B, Wu J, Sheng WT, et al. Comparative Analysis of Anther Transcriptome Profiles of Two Different Rice Male Sterile Lines Genotypes under Cold Stress. International Journal of Molecular ences. 2015;16(5):11398.

34. Shin JH, Yoshimoto K, Ohsumi Y, et al. OsATG10b, an autophagosome component, is needed for cell survival against oxidative stresses in rice. Molecules Cells. 2009;27(1):67-74.

35. Xia K, Liu T, Ouyang J, et al. Genome-Wide Identification, Classification, and Expression Analysis of Autophagy-Associated Gene Homologues in Rice (Oryza sativa L.). DNA Res. 2011;18:363-77.

36. Peleg Z, Blumwald E. Hormone balance and abiotic stress tolerance in crop plants. Curr Opin Plant Biol. 2011;14(3):290-5.

37. Kong X, Tian H, Ding Z. Plant Hormone Signaling Mediates Plant Growth Plasticity in Response to Metal Stress. Mechanism of Plant Hormone Signaling under Stress. 2017;223-235.

38. Giang LQ, Yuki IK, Kenji K, et al. Cadmium impairs protein folding in the endoplasmic reticulum and induces the unfolded protein response. Fems Yeast Research. 2016;(5):49.

39. Ammar WB, Nouairi I, Zarrouk M, et al. The effect of cadmium on lipid and fatty acid biosynthesis in tomato leaves. Biologia. 2008;63(1):86-93. 
40. Lin CY, Trinh NN, Fu SF, et al. Comparison of early transcriptome responses to copper and cadmium in rice roots. Plant Mol Biol. 2013;81(4-5):507-22.

41. Dubey S, Misra P, Dwivedi S, et al. Transcriptomic and metabolomic shifts in rice roots in response to $\mathrm{Cr}(\mathrm{VI})$ stress. BMC Genom. 2010;11(1):648-8.

42. Coombs JT, Franco CM. Visualization of an endophytic Streptomyces species in wheat seed. Appl Environ Microbiol. 2003;69:4260-2.

43. Chen C, Begcy K, Liu K, et al. Molecular characterization of rice endosperm development under heat stress identifies OsMADS87 as a determinant of seed size and thermal sensitivity. Plant Physiol. 2016;171(1):606-22.

44. Huang XY, Chao DY, Gao JP, et al. A previously unknown zinc finger protein, DST, regulates drought and salt tolerance in rice via stomatal aperture control. Genes Dev. 2009;23(15):1805-17.

\section{Figures}



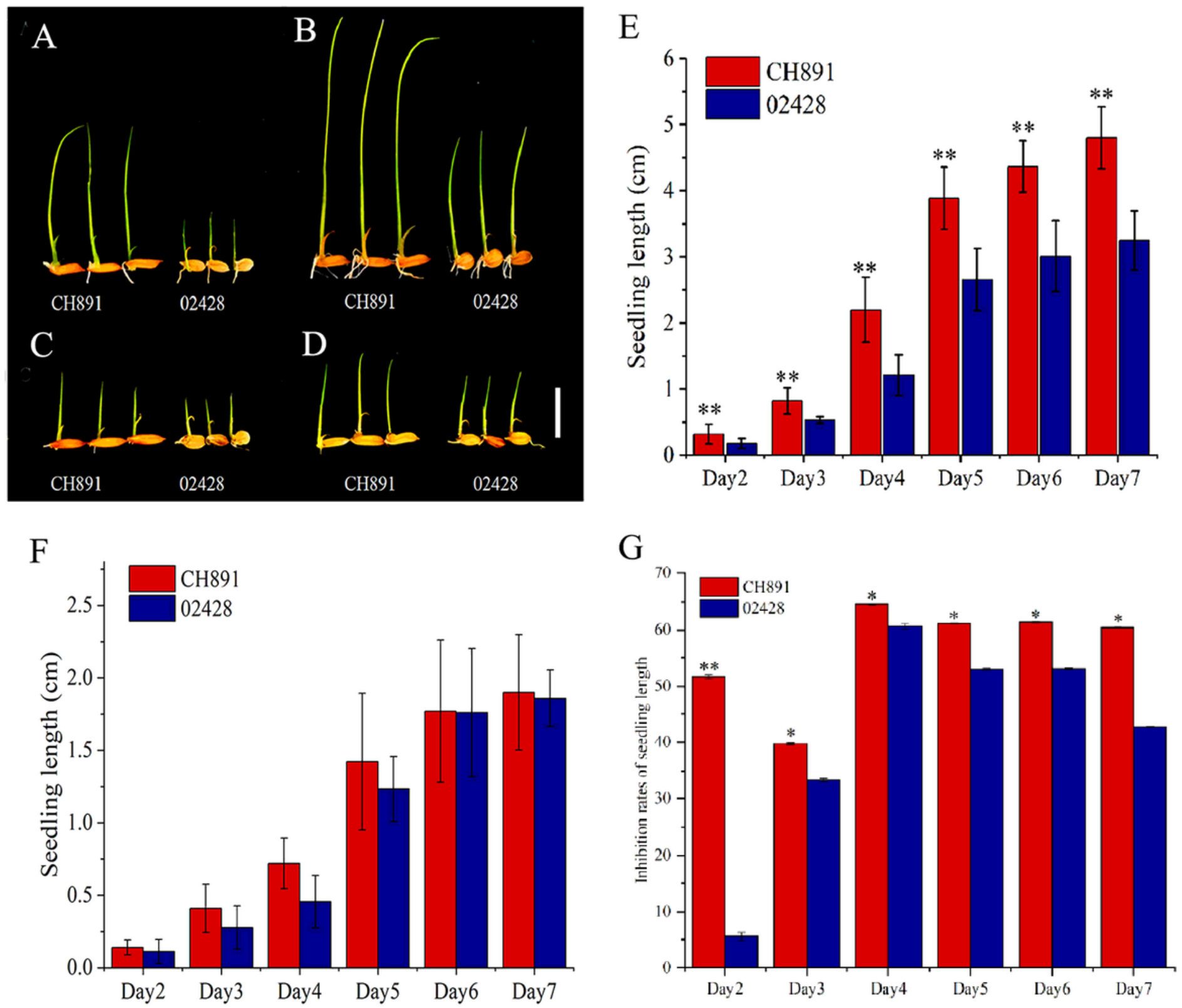

\section{Figure 1}

Phenotypes of $\mathrm{CH} 891$ and 02428 . (A) Left: $\mathrm{CH} 891$ under control condition at 3rd day. Right: 02428 under control condition at 3rd day. (B) Left: $\mathrm{CH} 891$ under control condition at 5th day. Right: 02428 under control condition at 5thday. (C) Left: CH891 under Cd stress at 3rd day. Right: 02428 under Cd stress at 3rd day. (D) Left: $\mathrm{CH} 891$ under Cd stress at 5th day. Right: 02428 under $\mathrm{Cd}$ stress at 5th day. Scale bar, 10 $\mathrm{mm}$. (E) The seedling length of $\mathrm{CH} 891$ and 02428 under control condition for six days in a row. ** highly significant difference. (F) The seedling length of $\mathrm{CH} 891$ and 02428 under $\mathrm{Cd}$ stress for six days. (G) The seedling length inhibition rate under $\mathrm{Cd}$ treatment compared with control group. 


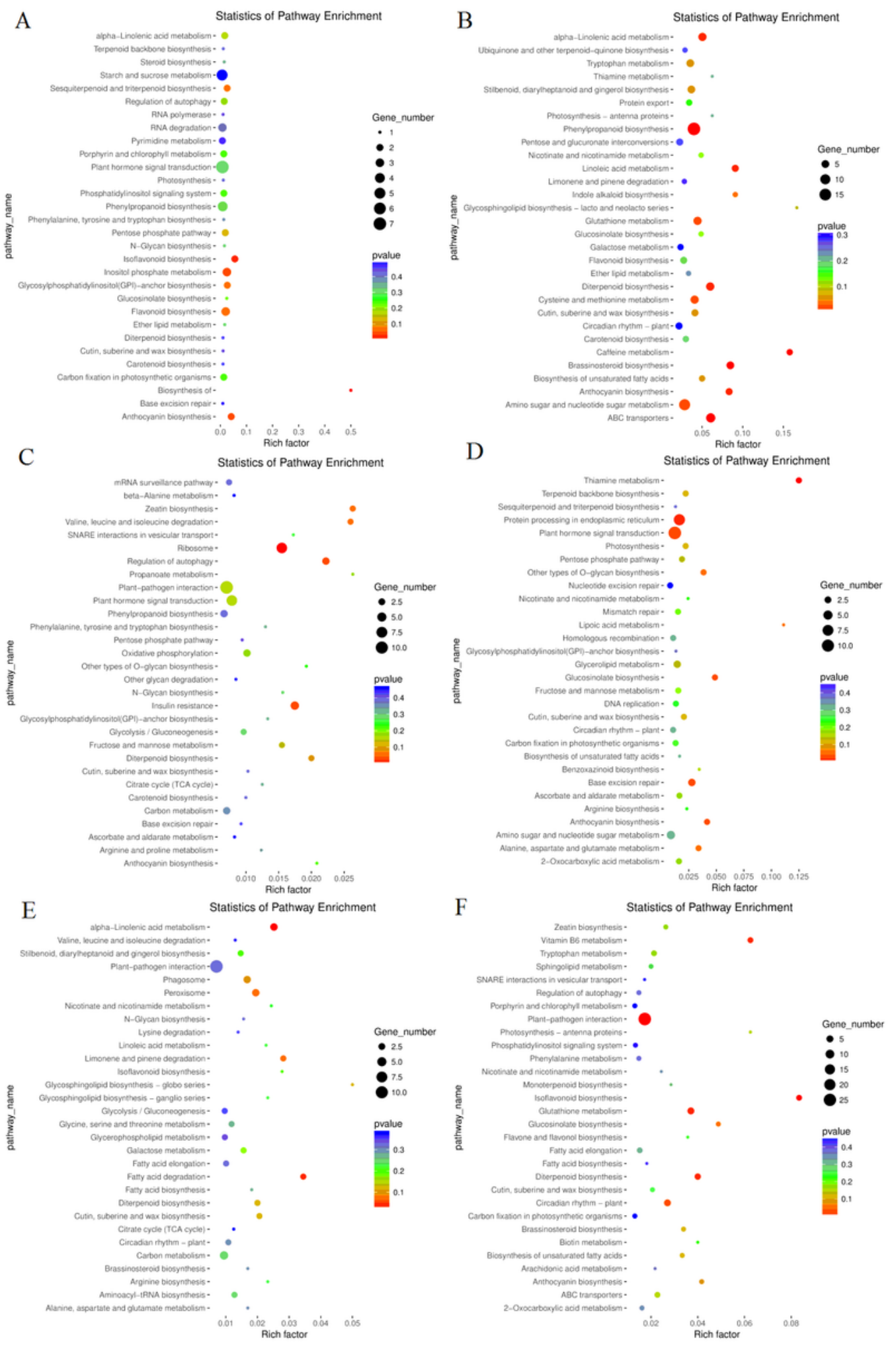

Figure 2

The KEGG terms of ICR genes from SCR, RCR and CCR. (A) SCR3, (B) SCR5, (C) RCR3, (D) RCR5, (E) CCR3, (F) CCR5. P-value means degree of enrichment, and its less value means greater intensiveness. 

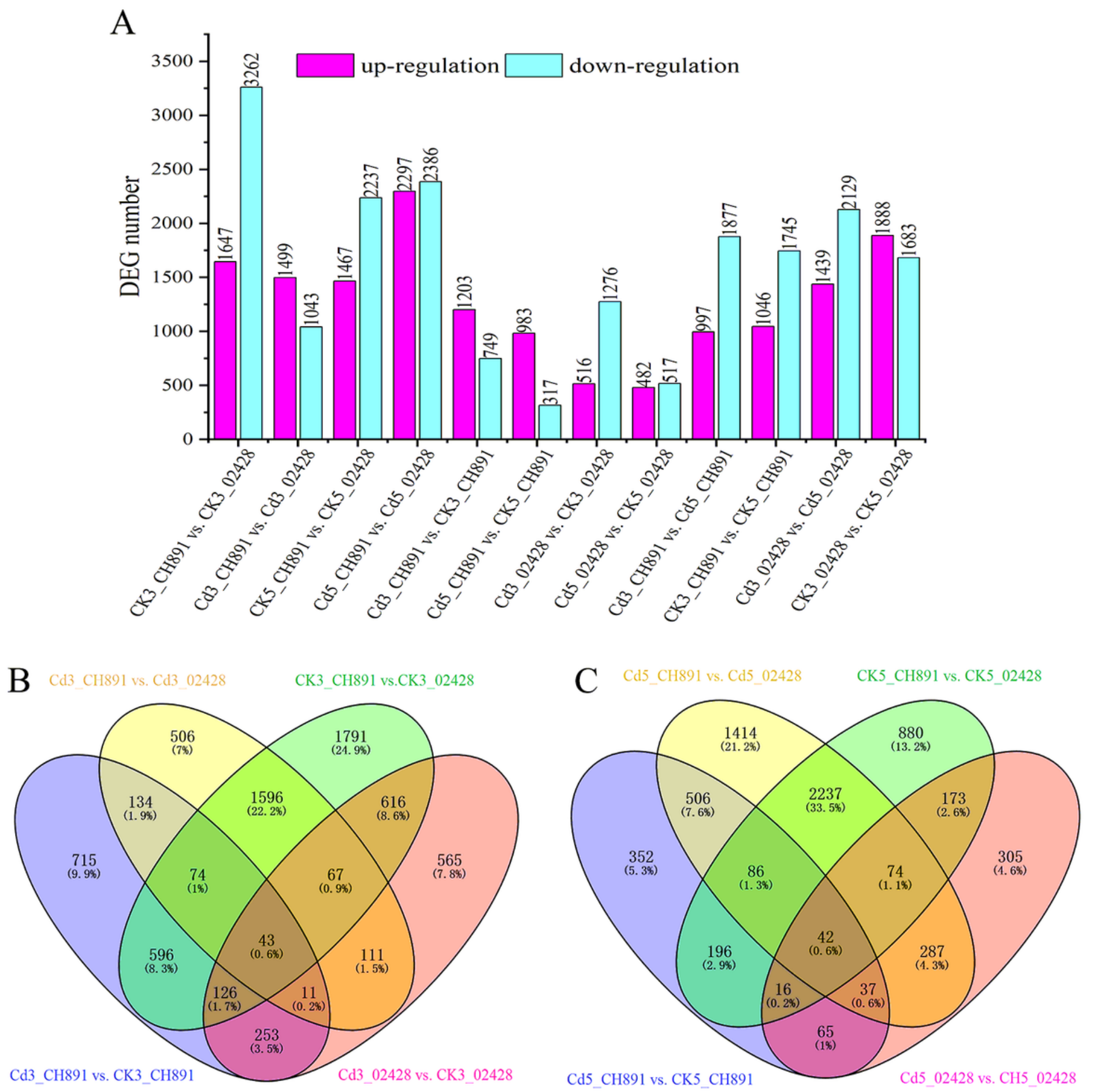

\section{Figure 3}

Identification of differentially expressed genes (DEGs) in the different comparison groups at 3rd and 5th day. (A) Up and down regulated gene numbers in eight comparison groups. (B) Venn diagrams for DEGs in the four comparison groups at 3rd day. (C) Venn diagrams for DEGs in the four comparison groups at 5th day. 


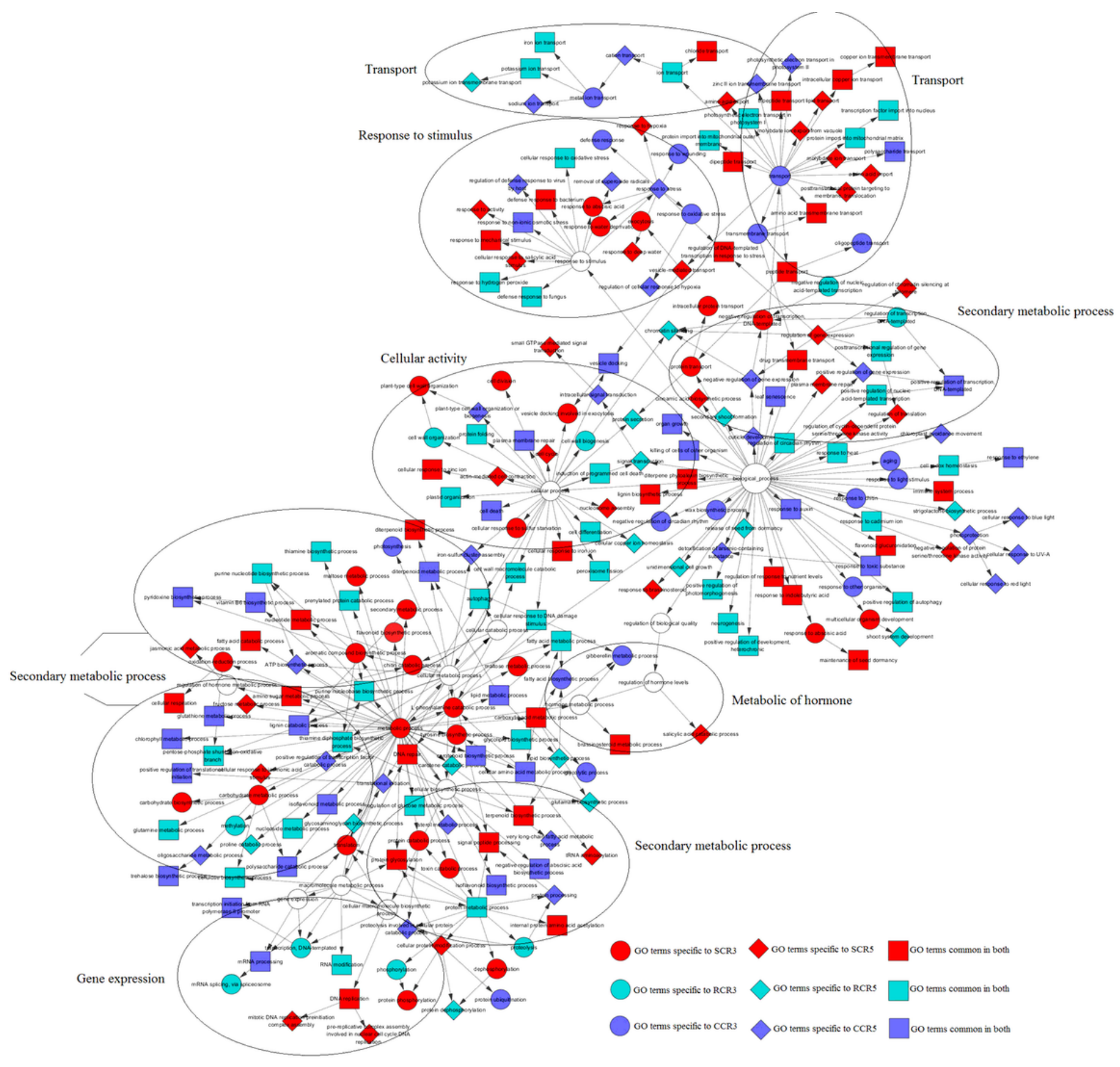

Figure 4

Network of significantly enriched GO terms (biological process) in SCR, RCR and CCR. 
A

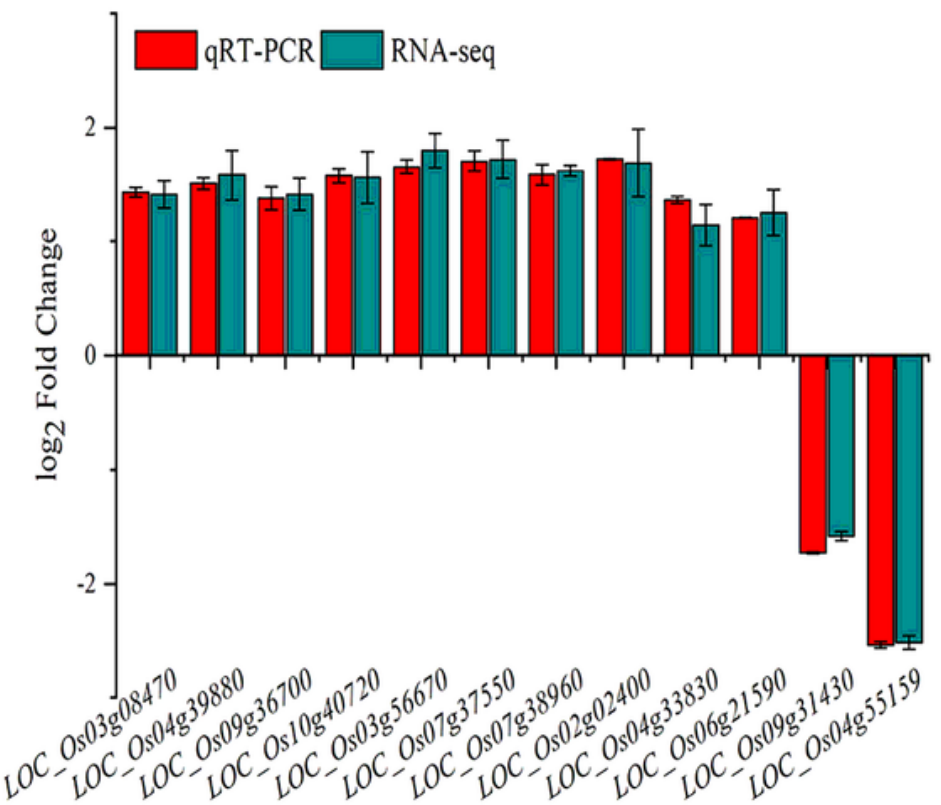

B

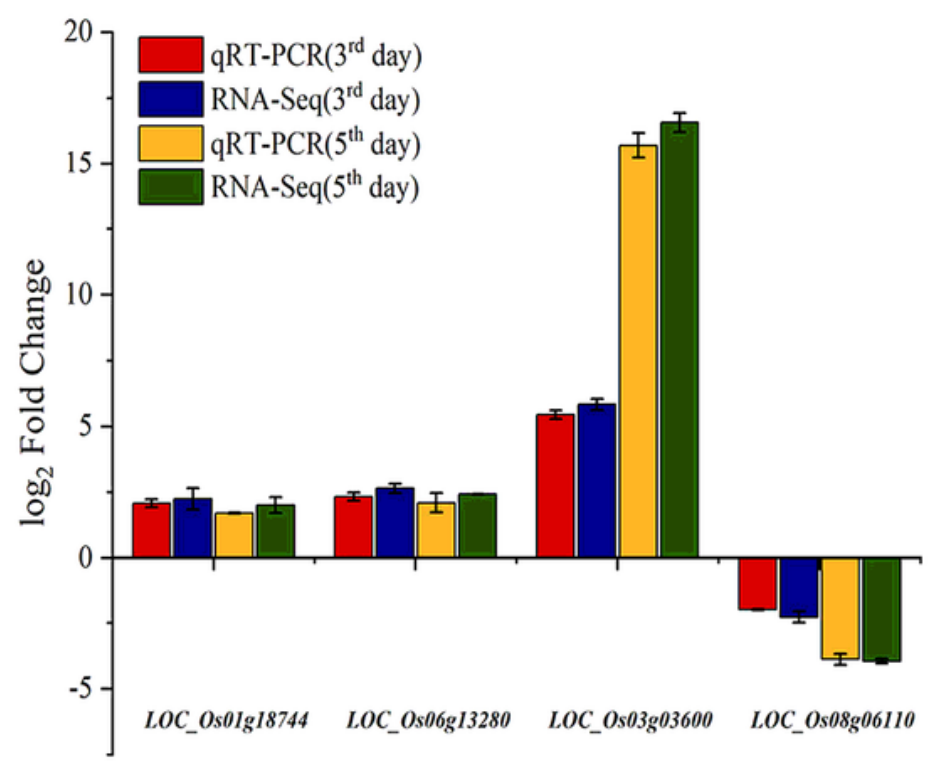

C

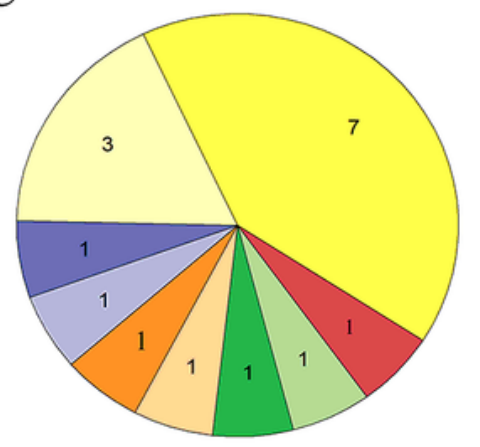

Cutin, suberine and wax biosynthesis

Protein processing in endoplasmic reticulum Inositol phosphate metabolism Phenylpropanoid biosynthesis

Isoflavonoid biosynthesis

Biosynthesis of amino acids

Ribosome biogenesis in eukaryotes

Plant hormone signal transduction

Unknown
$\mathrm{D}$

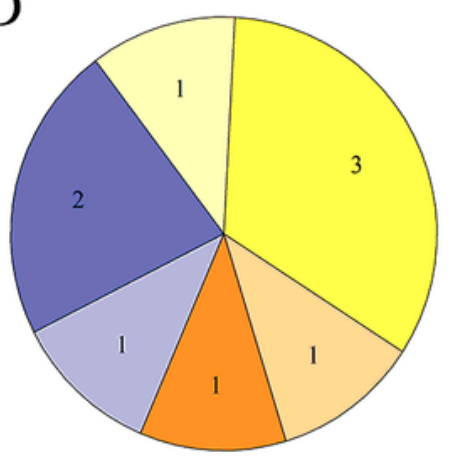

Ascorbate and aldarate metabolism

Starch and sucrose metabolism

Base excision repair

Plant hormone signal transduction

Protein processing in endoplasmic reticulum

Unknown
$\mathrm{E}$

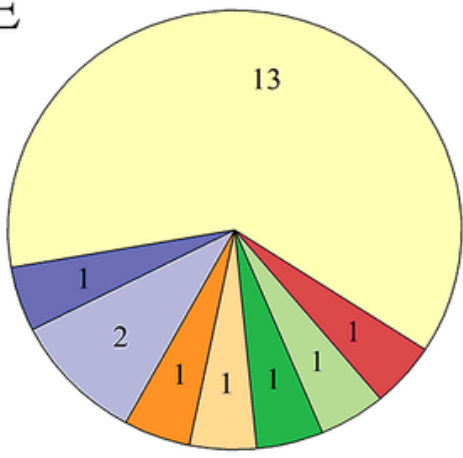

Galactose metabolism Fatty acid elongation Diterpenoid biosynthesis Brassinosteroid biosynthesis Aminoacyl-tRNA biosynthesis Plant-pathogen interaction Circadian rhythm - plant Unknown

Figure 5

The comparison of RNA-seq results and qRT-PCR analysis of gene expression levels and the KEGG pathways of common ICR in SCR, RCR, CCR at two stages. (A) LOC_Os03g08470, LOC_Os04g39880 and LOC_Os09g36700 were in Cd3_CH891 vs. CK3_CH891, LOC_Os10g40720 was in Cd3_02428 vs. CK3_02428, LOC_Os03g56670, LOC_0s07g37550 and LOC_Os07g38960 were in Cd5_02428 vs. CK5_02428, LOC_Os02g02400, LOC_Os04g33830, LOC_Os06g21590 and LOC_Os09g31430 were in 
Cd3_CH891 vs. Cd3_02428, LOC_Os04g55159 was in Cd5_CH891 vs. Cd5_02428. (B) LOC_Os01g18744 and LOC_Os06g13280 were in Cd3_CH891 vs. CK3_CH891 and Cd5_CH891 vs. CK5_CH891, LOC_Os03g03600 and LOC_Os08g06110 were in Cd3_CH891 vs. Cd3_02428 and Cd5_CH891 vs. Cd5_02428. (C) KEGG pathways of common ICR in SCR at two stages. (D) KEGG pathways of common ICR in RCR at two stages. (E) KEGG pathways of common ICR in CCR at two stages.

\section{Supplementary Files}

This is a list of supplementary files associated with this preprint. Click to download.

- SupplementaryTableS3.xlsx

- SupplementaryTableS2.xlsx

- SupplementaryTableS1.xlsx

- SupplementaryFiguresS1S2.pdf 